\title{
Periodontitis como factor de riesgo de enfermedades cardiovasculares
}

Periodontitis as a risk factor for heart diseases

Periodontite como fator de risco de cardiopatias
Fecha de Recepción

10 de abril de 2016
Aceptado para su publicación

I8 de diciembre de 2016

\section{Profesor de Periodoncia}

J. Monzón

(Facultad de Odontología-UNNE).

M. Acuña

Docente de Fisico-Quimica (FOUNNE)

C. Caramello

Docente de Periodoncia (FOUNNE)

J. Sesín

Adscripto de Periodoncia ( FOUNNE)

\section{Resumen}

Las enfermedades cardiovasculares representan una de las principales causas de muerte en las sociedades desarrolladas. Estas enfermedades no sólo son importantes por la mortalidad que generan, sino por la gran cantidad de individuos afectados y en tratamiento que han de soportar los sistemas de salud de los distintos países. De ahí que la detección precoz de los factores de riesgo conducentes a desencadenar estas enfermedades haya sido vista como el mecanismo más eficaz para prevenirlas. En los últimos tiempos se viene relacionando de una manera más o menos directa a la patología periodontal con el padecimiento de enfermedades cardiovasculares. Este hecho podría tener una gran relevancia clínica y probablemente epidemiológica, debido a la considerable prevalencia actual de las enfermedades periodontales. En la presente revisión se analizan los factores clínicos y fisiopatológicos que dan soporte científico a esta asociación, llegándose a la conclusión de que hacen falta más estudios, para tener la evidencia de que dicha asociación, representa un factor de riesgo determinante para el padecimiento de las enfermedades cardiovasculares.

\section{Palabras claves}

Cardiopatía, enfermedad coronaria, enfermedad periodontal, infarto agudo de miocardio, periodontitis. 
REVISTA FACULTAD DE ODONTOLOGÍA

ISSN No 1668-7280 - Vol. X No 1 - 2017

33
DIVULGACIÓN

Periodontitis como factor de riesgo de

enfermedades cardiovasculares

\section{Abstract}

Cardiovascular diseases represent one of the main causes of death in developed societies. These diseases are not only important by the mortality they generate, but because of the large number of individuals affected and in treatment that have to be supported by the health systems from different countries. Early detection of risk factors triggered to lead in these diseases has been seen as the most effective mechanism to prevent them. In the lasted times periodontal disease has been linked in a more or less direct way to cardiovascular disease. This fact could have a great clinical relevance, and probably epidemiological due to the actual considerable recent prevalence of periodontal diseases. In the present review clinical and pathophysiological factors are analyzed, in order to get a scientific support to this association, concluding that more studies are needed to have evidence that this association represents a determinat risk factor for the condition of cardiovascular diseases.

\section{Key Words}

Heart disease, coronary heart disease, periodontal disease, acute myocardial infarction, periodontitis.

\section{Resumo}

As doenças cardiovasculares são uma das principais causas de morte nas sociedades desenvolvidas. Estas doenças não são apenas importantes pela mortalidade que geram, mas também pelo grande número de indivíduos afetados e tratamentos complexos que exigem dos sistemas de saúde dos diferentes países. Daí, a detecção precoce dos fatores de risco que desencadeiam estas doenças tem sido visto como o mecanismo mais eficaz para preveni-las. Nos últimos tempos, as doenças cardiovasculares têm sido ligadas de forma relativamente direta à doença periodontal. Isso pode ter grande significado clínico e epidemiológico, provavelmente devido à atual prevalência considerável de doenças periodontais. Na presente revisão são analisados fatores clínicos e fisiopatológicos que dão apoio científico a esta associação, e concluíram que são necessários mais estudos para ter evidências de que esta associação representa um fator de risco significativo para a condição de doença cardiovascular.

\section{Palavras chaves}

Doença cardíaca, doença cardíaca coronária, doença periodontal, infarto agudo do miocárdio, periodontite.

\section{Introducción}

La periodontitis es una enfermedad infecciosa que afecta los tejidos de sostén de los dientes (ligamento periodontal, hueso alveolar y cemento radicular). Su etiología es multifactorial y su prevalencia se estima en el rango de 30 a $40 \%$ en la población adulta. Su patogenia resulta de la interacción entre un biofilm predominantemente gram negativo anaerobio y algunas especies microaerófilas y el sistema inmune del huésped. Su progresión en el tiempo sin tratamiento puede traer como consecuencia la movilidad y posterior pérdida de las piezas dentarias afectadas, junto la exposición del epitelio ulcerado de la bolsa (por la acción de colagenasas) a la placa bacteriana subgingival.El daño que se genera en el epitelio favorece el paso de bacterias hacia la circulación sanguínea, generando bacteriemias transitorias durante al menos el $40 \%$ de las veces que cepillamos nuestros dientes. Durante las bacteriemias se han encontrado más de 30 especies bacterianas, siendo predominantes los Streptoccocusviridans, responsables de patologías como fiebre reumática, valvulopatías y endocarditis bacteriana. La periodontitis puede provocar alteraciones hemostáticas, como el aumento del fibrinógeno plasmático, del recuento de glóbulos blancos, de la proteína $C$ reactiva y de la viscosidad de la sangre. Además se ha encontrado una relación del factor von Willebrand, o factor VIII (glicoproteína sintetizada por las células del endotelio vascular y megacariocitos), con el LPS y la IL-I, quienes inducen la liberación de este factor desde las células endoteliales, generando la agregación de plaquetas y focos inflamatorios donde se puede generar un trombo. La interrelación fisiopatológica entre ambas enfermedades, despierta un gran interés en controlar la periodontitis como un nuevo integrante en la lista de factores de riesgo para la enfermedad cardiovascular. 
REVISTA FACULTAD DE ODONTOLOGÍA

ISSN No 1668-7280 - Vol. X No 1 - 2017
DIVULGACIÓN

Monzón - Acuña - Caramello - Sesín

\section{Desarrollo}

Analizando específicamente las muertes por enfermedades del aparato circulatorio en varones, la cardiopatía isquémica es la causa más frecuente, seguida de cerca por la patología cerebrovascular y la enfermedad hipertensiva; en mujeres la cardiopatía isquémica es la segunda en importancia después de la patología cerebrovascular, ocupando igualmente el tercer lugar la enfermedad hipertensiva ${ }^{1,2,3}$. La cardiopatía isquémica es de origen multifactorial. Los factores que la predisponen deben cumplir el criterio de causalidad: fortaleza de asociación (alto riesgo relativo), consistencia de la asociación (demostrada en varios estudios), relación temporal (la causa precede al efecto), plausibilidad biológica, evidencia experimental y sobre todo, evidencia de estudios en humanos'. La hipertensión arterial (HTA) es, junto con el consumo de tabaco y la hipercolesterolemia, uno de los principales factores de riesgo para el desarrollo de la cardiopatía isquémica y el principal factor de riesgo para los accidentes cerebrovasculares (ACV). Además, es causa frecuente de insuficiencia cardiaca en el adulto y de otras enfermedades cardiovasculares, como aneurisma disecante de aorta ${ }^{3,4}$. Asimismo, el riesgo de enfermedad cardiovascular en pacientes hipertensos es tres veces superior al de no hipertensos; existe un riesgo 2 a 5 veces superior de padecer cardiopatía isquémica y 7 veces superior de padecer enfermedad cardiovascular, por lo que en este caso puede hablarse de un factor de riesgo de un elevado grado de asociación ${ }^{5,6}$. $\mathrm{Ha}$ tenido que pasar demasiado tiempo para comprender que la boca no es una estructura aislada dentro del conjunto del organismo, y aunque se conoce la importancia de la cavidad oral en el campo de la fisiología humana y en determinados grupos de enfermedades, como puede ser el cáncer oral, poco se sabe sobre la relación de las infecciones propias de los tejidos dentales y periodontales con otros órganos o sistemas del cuerpo humano ${ }^{7}$. La pregunta sería, ¿es posible que en un paciente sano con una enfermedad infecciosa oral de carácter crónico se pueda producir un efecto perjudicial en su organismo como consecuencia de la agresión bacteriana continuada? Esta pregunta es ciertamente importante, y la respuesta afirmativa a la misma podría modificar la actitud de los pacientes, los médicos y los odontólogos hacia este tipo de enfermedades ${ }^{7,8}$. Las enfermedades periodontales están condicionadas en su inicio, desarrollo y gravedad por una serie de factores generalmente conocidos. Ahora bien, actualmente se destaca el posible papel de la patología periodontal en la evolución de determinadas enfermedades sistémicas, fundamentalmente aquellas derivadas de procesos isquémicos cardiovasculares ${ }^{7,8}$. Existe una estrecha relación entre los procesos inflamatorios y la aterosclerosis, como lo prueba el hecho de que factores como la hipertensión, la diabetes, el colesterol o la obesidad, que incrementan el tono inflamatorio del cuerpo, están muy relacionados con el riesgo cardiovascular. La explicación de este incremento del riesgo en un plazo largo en el tiempo se encuentra en el hecho de que la enfermedad coronaria es un proceso multifactorial, en el que los mecanismos inflamatorios juegan un papel importante, pues los factores de riesgo convencionales, las infecciones crónicas y la producción de radicales libres de oxígeno elevan los niveles de citosinas. Estas pasan a la sangre, actúan sobre otras células y el resultado final es el daño del vaso ${ }^{8}$. Cuando se analiza la relación que puede existir entre dos grupos de procesos tan diferentes, es lógico descartar todo aquello que esté relacionado con la clínica, el diagnóstico, el tratamiento y el pronóstico de los mismos, ya que es evidente lo poco que tienen en común. Si la relación es de causa y efecto, el mecanismo común que pueda unir a estas dos enfermedades habrá que buscarlo en el ámbito etiopatogénico? Analizando las bases actuales de la etiología de las enfermedades periodontales, está fuera de duda el papel tan importante que juegan las bacterias gram-negativas en el inicio y progresión de las mismas ${ }^{8,9}$. También sabemos que estos microorganismos, o los productos derivados de los mismos, pueden acceder al sistema vascular del tejido conectivo subyacente, y de ahí pasar a la circulación general, proporcionando unas posibilidades muy elevadas de intercambio de productos tóxicos bacterianos entre la bolsa periodontal y el sistema circulatorio. Esta situación no pasa inadvertida para el organismo, pudiendo generar una respuesta inmune superior a lo esperado. Baste como ejemplo saber que la leucotoxina del Actinobacillusactinomycetemcomitans 
REVISTA FACULTAD DE ODONTOLOGÍA

ISSN No 1668-7280 - Vol. X No 1 - 2017

35
DIVULGACION

Periodontitis como factor de riesgo de

enfermedades cardiovasculares provoca una respuesta de anticuerpos con unos niveles séricos similares a los que se pueden detectar en algunas enfermedades sistémicas diseminadas, como la sífilis terciaria ${ }^{10,11}$. Actualmente se piensa que el factor común más probable entre ambas enfermedades es la interacción entre los productos de la placa bacteriana y las respuestas inflamatoria e inmunológica del huésped $^{12}$. Como ya se dijo anteriormente, como consecuencia de la agresión bacteriana en la enfermedad periodontal, se liberan por parte de las células de defensa una serie de mediadores de la inflamación, entre los que destacan la interleucina I (IL-I), el factor de necrosis tumoral (TFN) y el tromboxano A2. Estas citoquinas, independientemente del efecto lesivo que producen en los tejidos periodontales, pueden estimular la adhesión y la agregación plaquetaria, promover el acumulo de células espumosas cargadas de lípidos y la deposición de colesterol en la íntima vascular. Además, estas citoquinas asociadas a la acción del factor de crecimiento derivado de las plaquetas (PDGF) estimulan la proliferación de la musculatura lisa vascular, favoreciendo el estrechamiento de la luz de los vasos y promoviendo a su vez la formación de la placa de ateroma ${ }^{12,13}$. La respuesta a la agresión de las bacterias periodontopatógenas es muy variable de unos individuos a otros ${ }^{14}$. Estas diferencias deben residir en el sistema de defensa del huésped, de tal manera que algunas personas responden a la presencia de determinadas bacterias y a los lipopolisacáridos de las mismas con una reacción inflamatoria exagerada, caracterizada por la secreción por parte de sus monocitos de unos niveles muy elevados de los mediadores descritos previamente. Estos individuos segregan estas sustancias en concentraciones superiores a los individuos normales, teniendo losmediadores un papel fundamental en la patogénesis de la destrucción periodontal ${ }^{15}$. Además, los monocitos juegan un papel importante en el desarrollo de la placa de ateroma, y pueden justificar una base biológica que una a las enfermedades periodontales y a la patología coronaria, pudiendo actuar como mecanismo desencadenante común la acción de los lipopolisacáridos bacterianos ${ }^{15,16}$. La presencia de periodontitis debe ser considerada como un factor de riesgo para desarrollar una enfermedad cardiovascular $y$, por lo tanto, se aconseja su preven- ción y tratamiento ${ }^{17}$. Así se contempla en la versión 2014, recientemente publicada en el European Heart Journal, de las guías promovidas por la Sociedad Europea de Cardiología (SEC) para la prevención de la enfermedad cardiovascular en la práctica clínica. Estas directrices proceden de los trabajos efectuados por la $5^{\text {a Join }}$ Task Force de la Sociedad Europea de Cardiología y otras sociedades científicas afines relacionadas con la prevención de la enfermedad cardiovascularl, ${ }^{18}$. Aunque ya se tenían numerosas evidencias científicas y clínicas sobre el impacto negativo que tiene la periodontitis en el ámbito cardiovascular y sobre el aumento de aparición de eventos cardiovasculares en personas con periodontitis, esta recomendación de la SEC supone un espaldarazo definitivo para la estrategia de prevención y manejo de las infecciones de las encías, con el objetivo de evitar consecuencias nefastas a nivel cardiovascular. En concreto, en las guías se advierte expresamente que la periodontitis se asocia con la aparición de disfunción endotelial, aterosclerosis y con un incremento del riesgo de infarto de miocardio', 19, 20. De la misma forma, se reconoce que otros factores, como el bajo nivel socioeconómico y el hábito tabáquico, también pueden tener una importante influencia (y actuar como factores de confusión) en esta relación entre periodontitis y enfermedades cardiovasculares ${ }^{21,22,23}$. Por todo ello, en las Guías de la SEC se subraya que "la periodontitis puede ser considerada como un indicador de riesgo para alcanzar un bajo estatus de salud cardiovascular" y, por eso, se afirma que "su tratamiento está indicado de la misma forma que lo está el manejo de los otros factores de riesgo cardiovascular subyacentes" $24,25,26$. En estas guías, la periodontitis se sitúa en un apartado junto a otros trastornos de reconocido impacto negativo en la esfera cardiovascular, como la enfermedad renal crónica, la apnea del sueño, las enfermedades autoinmunes, la gripe o la disfunción eréctil ${ }^{27,28}$. Cardiólogos y odontólogos coinciden en asegurar ya que las enfermedades que afectan a las encías (especialmente las infecciones periodontales) son un factor de riesgo para sufrir episodios coronarios, algunos tan graves que pueden llegar a poner en peligro la propia vida del paciente (29-30). 


\section{Conclusión}

La enfermedad periodontal puede estar relacionada con la enfermedad cardíaca, ya que la boca no es un ente aislado de los otros órganos y sistemas. Si bien, el factor de riesgo más importante para el desarrollo de enfermedades cardíacas es el genético existen bacterias potencialmente periodontopáticas como la P. gingivalis y otras que son capaces de invadir intracelularmente las células endoteliales y producir un daño directo evadiendo la respuesta inmune del huésped. Tanto los microorganismos como sus productos producen IL-6 y CRP, que entran a la circulación y contribuyen al desarrollo de los ateromas. Los niveles séricos de CRP e IL-6 se reducen de manera significativa con el tratamiento periodontal. Los niveles de CRP séricos están directamente vinculados con la severidad de la enfermedad periodontal, es decir, mientras más severa sea la enfermedad periodontal, más elevados son los valores de CRP séricos. El daño producido por las bacterias puede ser directo $o$ indirecto; el segundo se encuentra vinculado con el factor genético, ya que hay personas que poseen una predisposición genética para tener una respuesta inmune exagerada y una mayor producción de citoquinas inflamatorias.

\section{Bibliografía}

I. Barrios V, Soria F, Placer L, Cruz JM, Tomás L, Rodríguez $L$ et al. Guías de práctica clínica de la Sociedad Europea de Cardiología en hipertensión arterial. Rev Esp de Cardiol 2014;53: 66-90.

2. Ebersole JL. Systemic humoral immune responses in periodontal diseases.CritRev Oral BiolMed 2006; I:283-33।.

3. Guadalajara JF. Cardiología. En: Martín L, ed. PAC MG-I Programa de actualización continua para médicos generales. Academia Nacional de Medicina. México: Méndez Editores;20I I. p.43-8.

4. Haffajee AD, Sockransky SS. Microbial etiology of destructive periodontal disease.An overview. Periodontology 2000 2002; 5:78-III.

5. Hakim AA, Curb JD, Petrovitch H, Rodríguez BL, Yano K, Ross GW et al. Effects of walking on coronary heart disease in elderly men: the Honolulu Heart Program. Circulation 2010;100:9-13.

6. Hernichel-Gorbach E, Kornman KS, Holt SC, Nichols F, Meador H, Kung JT et al. Host responses in patients with generalized refractory periodontitis. J Periodontol 20I 0;65:8-I6.

7. Herzberg MC, Meyer MW. Effects of oral flora on platelets: possible consequences in cardiovascular disease. J Periodontol 201 I; 67: II38-42.

8. Hienz SA, Paliwal S, Ivanovski S. Mechanisms of Bone Resorption in Periodontitis. J Immunol Res, vol. 2015, Article ID 615486, 10 pages, 2015. doi: 10.1 I55/20I5/6I5486.

9. Hofbauer LC, Khosla S, Dunstan CR, Lacey DL, Boyle WJ, Riggs BL. The roles of osteoprotegerin and osteoprotegerin ligand in the paracrine regulation of bone resorption. J Bone Miner Res. 2000; I5(I):2-I2.

10. Holmlund A, Hänström L, Lerner UH. Bone resorbing activity and cytokine levels in gingival crevicular fluid before and after treatment of periodontal disease.J ClinPeriodontol. 2004, 3 I (6):475-482.

II. Kamma J, Mombelli A, Tsinidou K. Cytokines in gingival crevicular fluid of adolescents and young adults. Oral Microbiollmmunol. 2009;24(I):7-I0.

12. Kayal, R. A. (20I3). The Role of Osteoimmunology in Periodontal Disease.BioMed Research International, 2013, 639368.

13. Kneider M, Lowe CD, Murrav GD, Kinane DF, McGowan DA. Dental disease, fibrinogen and white cell count, link with myocardial infarction. Scot Med J 20II;38:73-4

14. Kornman KS, Pankow J, Offenbacher S, Beck J, Di Giovine F. Interleukin-I genotypes and the association between periodontitis and cardiovascular disease. J Periodontol Res 201 I;34:353-7.

15. Koshi E, Rajesh S, Koshi P, Arunima PR. Risk assessment for periodontal disease. J Indian SocPeriodontol. 2012; 16(3):324-328.

16. Kumar, A. K., Reddy, N. R., Babu, M., Kumar, P. M., Reddy, V. S., \&Chavan, C. V. (20I3). Estimation of 
prostaglandin E2 levels in gingival crevicular fluid in periodontal health, disease and after treatment. Contemporary Clinical Dentistry, 4(3), 303-306.

17. Leibur E, Tuhkanen A, Pintson U, Söder PO. Prostaglandin E2 levels in blood plasma and in crevicular fluid of advanced periodontitis patients before and after surgical therapy. Oral Dis. 1999;5:223-8.

18. Liu D, Xu JK, Figliomeni L, Huang L, Pavlos NJ, Rogers $M$, Tan A, Price P, Zheng $\mathrm{MH}$. Expression of RANKL and OPG mRNA in periodontal disease: possible involvement in bone destruction. Int] Mol Med. 2003; I I (I): I7-2I.

19. Liu S, Zhu W, Li S, Ma J, Zhang H, Li Z, Zhang L, Zhang B, Li Z, Liang X, Shi W. Bovine parathyroid hormone enhances osteoclast bone resorption by modulating V-ATPase through PTHIR. Int J Mol Med. 2016, 37(2), 284-292.

20. Marcus AJ, Hajar DR. Vascular transcellularsignalling. J Lipid Res 2010;34:2017-31.

2I. Mattila KJ, Valle MS, Nieminem MS, Valtonen W, Hietaniemi KL. Dental infections and coronary atherosclerosis. Atherosclerosis 2008; 103:205-I I.

22. Markou, E., Eleana, B., Lazaros, T., \&Antonios, K. (2009). The Influence of Sex Steroid Hormones on Gingiva of Women. The Open Dentistry Journal, 3, I I4-I 19. http://

23. Miyagi M, Morishita M, Mwamoto Y. Effects of sex hormones on production of prostaglandin E2 by human peripheral monocytes. J Periodontol. 1993;64: 1075-8.

24. Navarro F, de Teresa E, López-Sendon JL, Castro A. Guias del diagnóstico, clasificación y tratamiento de la insuficiencia cardiaca y del shock cardiogénico. Rev EspCardiol 2002; 52:1-54.

25. Offenbacher S, Heasman PA, Collins JG. Modulation of host PG-E2 secretion as a determinant of periodontal disease expression.J Periodontol 2002;64:432-44.

26. Rose LF, Mealey B, Minsk L, Cohen W. Oral care for patients with cardiovascular disease and stroke. JADA 2002; I33: 37-44.27. Scannapieco FA, Brush RB, Pau $\mathrm{S}$. Asociaciones entre enfermedad periodontal y el riesgo de aterosclerosis, enfermedad cardiovascular y apoplejía. Un estudio sistemático. Ann periodontol. 2003;8:38-53.

27. Seymour RA, Steele JG. Is there a link between periodontal disease and coronary heart disease?. Br Dent J 2009; 184: 33-8.

28. Shapira L, Soskolne WA, Sela MN, Offenbacher S, Barak V. The secretion of PG E2, IL-I, IL-6, TNF-alfa by monocytes from early onset periodontitis patients. J Periodontol 2009; 65: I39-46.

29. Scannapieco FA, Brush RB, Pau S. Asociaciones entre enfermedad periodontal y el riesgo de aterosclerosis, enfermedad cardiovascular y apoplejía. Un estudio sistemático. Ann periodontol. 2003;8:38-53.

30. Vettore MB. Enfermedad periodontal y enfermedad cardiovascular. Evid Based Dent. 2004;5: 69. 